\title{
Unusual rare case of upper gastrointestinal bleeding
}

\begin{abstract}
A case report of a 64-year-old man who presented with upper gastrointestinal bleeding due to Choledocholithiasis eroding to the duodenum, diagnosed with gastroscopy and a CT scan. The patient was managed by Choledocho-Jejunal anastomosis, after extraction of a giant common bile duct stone measuring $11.9 \times 5.0 \times 4.0$ centimeters.
\end{abstract}

Keywords: bile duct, biliary stone erosion, choledocholithiasis, gastrointestinal bleeding

\section{Introduction}

Bile duct stones obstructing the common bile duct may lead to complications such as pancreatitis and acute cholangitis. Though some patients may remain asymptomatic, others develop symptoms. Laboratory findings show Cholestatic liver function tests abnormality, with elevated serum Gamma-Glutamyltranspeptidase (GGT) and alkaline phosphatase. Impacted stones in the ampulla of vater may be removed with sphincterotomy; however, size, site and the form of the impacted stone will play a role in the difficulty of endoscopic treatment. In the case of large stones or multiple stones, recurrence is frequent. Surgical options will vary according to the clinical condition and the size of the dilated common bile duct.

Hemobilia, from the Greek haima (blood) and the Latin bilis (bile), occurs when an abnormal communication between the bile ducts and the blood vessels exists, causing hemorrhage in the bile ducts; While Bilhemia is a rare condition when bile enters the bloodstream.

Causes of hemobilia may be iatrogenic, traumatic, due to vascular diseases, neoplasms, inflammation, and gallstones. Microscopic hemorrhage occurs in a quarter of cases with cholelithiasis and in one-third of cases of Choledocholithiasis. Macroscopic Hemobilia is rare, it occurs in less than $1 \%$ of reported cases of hemobilia. It arises generally when a large stone erodes the cystic artery or enters into an adjacent vessel, or organ. Occasionally it can be the cause of a profuse Hemobilia; which must be regarded as a serious condition.

\section{Case Report}

A 77-year-old gentleman was admitted with a one-week history of melena, epigastric discomfort, postprandial abdominal pain, and coffee-ground emesis. Past Medical history hypertension and controlled diabetes mellitus; Past Surgical history open cholecystectomy ten years ago.

Physical exam revealed an ill-appearing patient hemodynamically stable, with tenderness in the right upper quadrant. Complete blood count showed no abnormalities; Hemoglobin -12.6, Y GT -585 UI/L, SGOT -135 UI/L, Alkaline phosphatase $-516 \mathrm{UI} / \mathrm{L}$, but normal bilirubin, amylase, and lipase; CRP was significantly elevated $(>150 \mathrm{mg} / \mathrm{L})$.

An Esophagogastroduodenoscopy (EGD) showed bleeding from an ulcer of the posterior duodenal wall, managed with the application of 2 clips. Two hours after the endoscopy, patient developed severe abdominal pain with repetitive episodes of hematemesis. An urgent abdominal CT (with iv contrast) FIGURES 1 and 2 revealed intrahepatic stones in segments 4 and 5 , large impacted common bile duct stone causing common bile duct fusiform dilatation with markedly dilated Intrahepatic and extrahepatic bile ducts. Thus repeat upper GI endoscopy was performed that confirmed bleeding from the previously described ulceration.

\section{Georges Khalifeh ${ }^{1}$, Nasr Bou Orm ${ }^{1}$, Richard Ghandour ${ }^{1}$, Ghassan J Awar ${ }^{1}$, Samer Dbouk ${ }^{1}$, Hussein Mcheimeche ${ }^{1}$ and Riad Sahili²}

${ }^{1}$ Department of General Surgery, AlZahraa Hospital, University Medical Center, Lebanon

${ }^{2}$ Department of General Surgery and Laparoscopic Bariatric surgery, AlZahraa Hospital, University Medical Center, Lebanon

*Author for correspondence: riad.sahili@zhumc.org.lb 

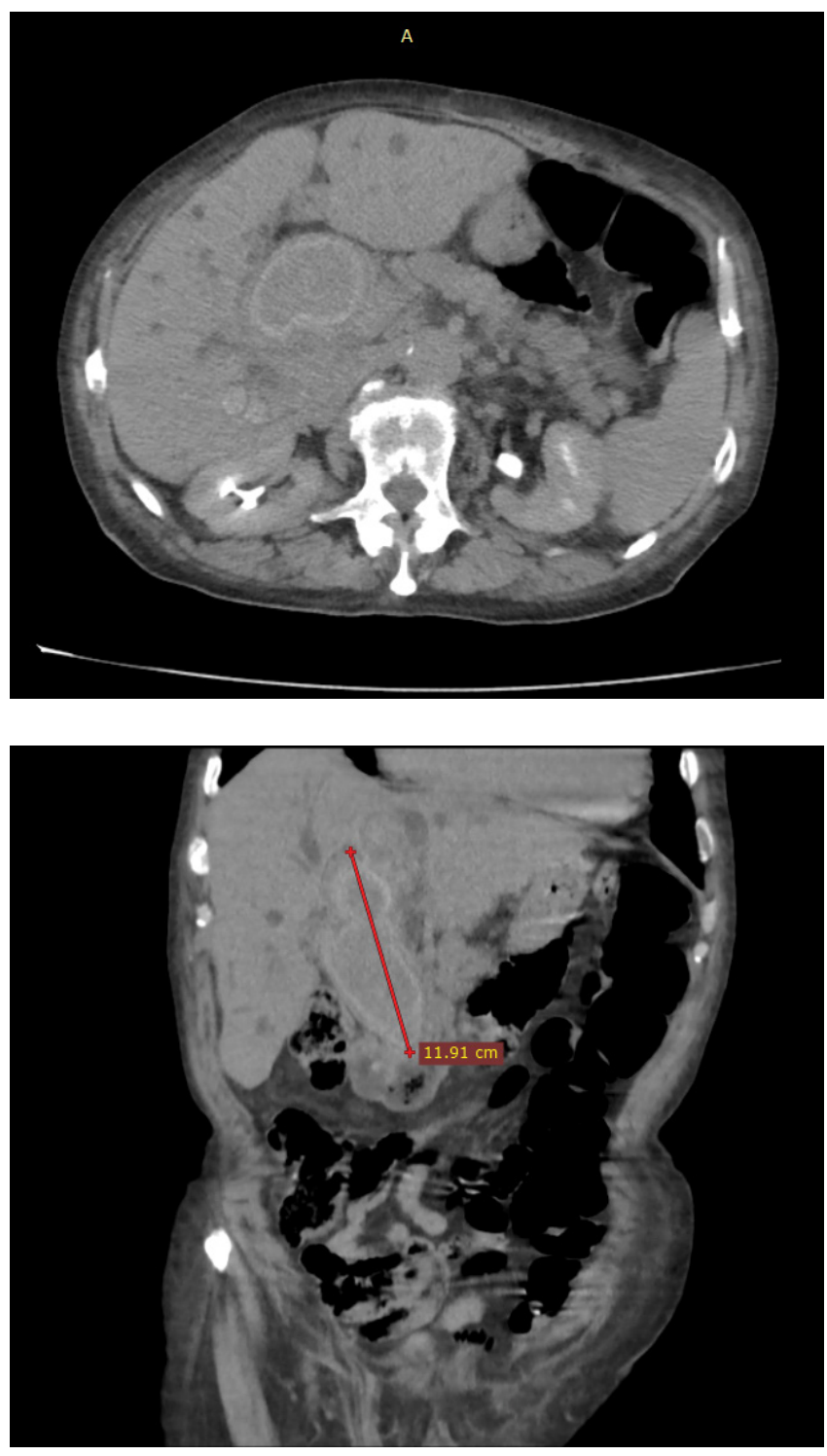

FIGURE 1. Computed tomography shows huge common bile duct stone of $5 \mathrm{~cm}$ in diameter impacted in the common bile duct.
FIGURE 2. Computed tomography shows huge common bile duct stone of $11.91 \mathrm{~cm}$ length bifurcated to right and left hepatic duct.
Due to the early recurrence and continuing bleeding; an exploratory laparotomy was decided. Upon exploration, multiple adhesions were found, that were carefully lysed. A longitudinal pyloromyotomy was done for better assessment of the bleeding source which seemed to be coming from the base of the ulcer. However the base of the ulcer was hard and it leads to communication with the common bile duct which was thickened and dilated. A palpable hard mass was felt in the middle portion of the CBD extending down to the duodenum.

A choledochotomy was done, revealing an enormous large stone $12 \times 5 \mathrm{~cm}$ (taking the shape of the CBD) and other multiple small stones filling the common bile duct. The giant stone was fragmented and removed in pieces and the intrahepatic stones were also cleared FIGURE 3. Atherectomy, Roux-en-Y Choledocho-
Enterostomy, and Gastro-Jejunostomy were performed as described in FIGURE 4. The post-operative course was complicated with bile fistula that closed spontaneously progressively after 2 weeks.

\section{Discussion and Conclusion}

In summary, patient with common bile duct stone, hemodynamically stable presented with severe hemobilia (major upper GI bleeding).

Stones in CBD may reach very considerable dimensions without causing serious symptoms. The most common symptom is jaundice. During the preoperative radiological examination, giant stones may be confused with malignancies [1]. Therefore, surgeons should follow conventional algorithms in diagnosis, and open surgery must be kept in mind in the earlier stages without relying totally on endoscopic interventions [2]. 
FIGURE 3. Fragments of stone extracted.

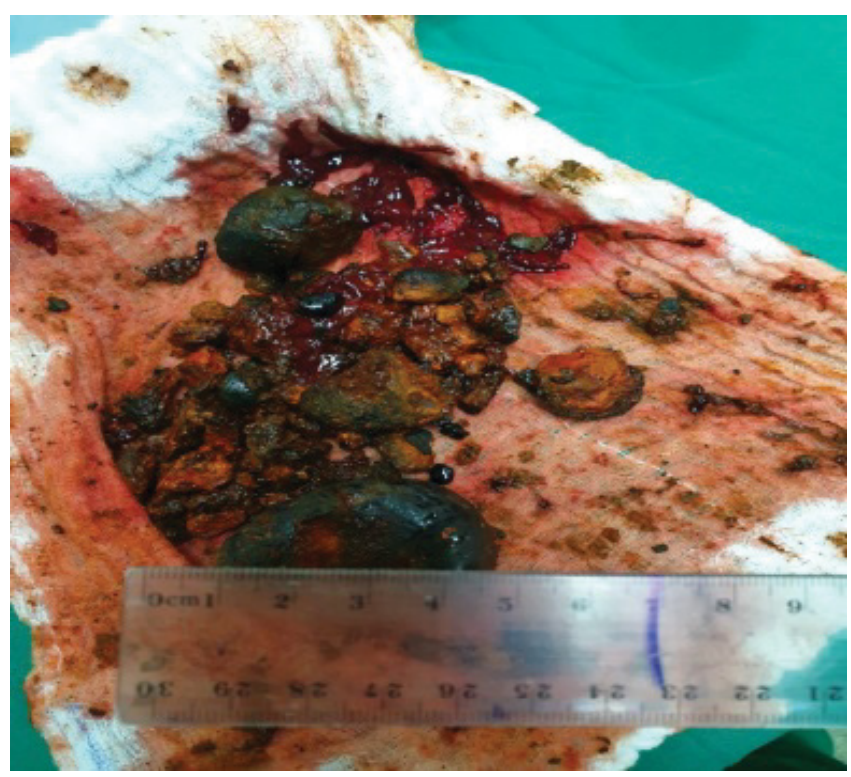

FIGURE 4. Choledocho-jejunostomy, gastrojejunostomy in roux en $\mathrm{Y}$ anastomosis.

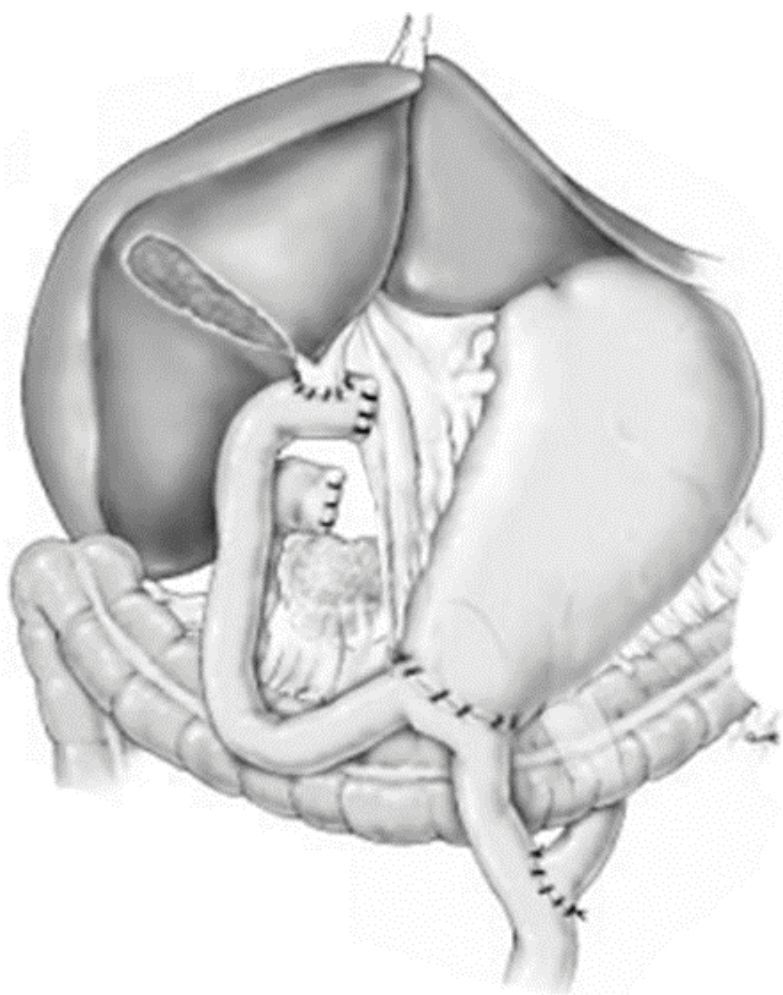

Several cases involving giant common duct stones have been reported, and hepatolithiasis was often associated. A case report dating back to 2012, describes gallstone-induced auto-sphincterotomy and resulting upper GI bleeding, similar to our case [3].

Giant common duct stones cause an insidious enlargement of ducts over time, with late symptoms. Undiagnosed "choledochal cyst" is a cause, especially when there is associated pancreaticobiliary junction anomaly. leads of common duct stones cause microscopic hemorrhage; however, macroscopic hemobilia is uncommon [4]. It generally occurs when large stones erode the cystic artery or penetrate into an adjacent viscous or vessel [5]. Chronic obstruction of the pancreatobiliary tract can potentially lead to inflammation, erosion, and fistulization with adjacent vascular structures with resultant hemobilia. Mechanical lithotripsy is reserved for stones smaller than $2.5 \mathrm{~cm}$, 
unlike our patient [6]. The initial diagnostic test of choice for patients with UGI bleeding is EGD, which might identify blood or clot at the ampulla of Vater in the setting of hemobilia. Selective arteriography of the hepatic artery is the best confirming test $[7,8]$. In our case, bleeding was not minor and hemostasis couldn't be achieved by therapeutic endoscopy $[9,10]$.

As for the surgical treatment, which mainly resides in ligation of the bleeding vessel, its use has decreased with the advent of non-operative modalities such as trans-arterial embolization $[11,12]$. Nevertheless, surgery remains the optimal option in patients with failed embolization or those in whom the etiology for hemobilia demands a surgical approach [11]. Gallstones, notably giant ones, are certainly on the list.

\section{Disclosure of Interest}

The authors declare that they have no competing interests. 


\section{References}

Abdelfatah MM, Mudireddy PR. Hemobilia: A rare cause of intermittent biliary obstruction. VideoGIE. 3, 236-237 (2018).

Steiner Z, Brown RA, Jamieson DH, et al. Management of hemobilia and persistent biliary fistula after blunt liver trauma. J Pediatr Surg. 29, 1575-1577 (1994).

Kalipershad S, Chung KT, Jehangir E. Upper gastrointestinal bleeding: Gallstone-induced auto-sphincterotomy. BMJ Case Rep. bcr2012006660 (2012).

Mays ET, Conti S, Fallahzadeh H, et al. Hepatic artery ligation. Surgery. 86, 536-543 (1979).
Abbas MA, Fowl RJ, Stone WM, et al. Hepatic artery aneurysm: Factors that predict complications. J Vasc Surg. 38, 4145 (2003).

Riemann JF, Seuberth K, Demling L. Clinical application of a new mechanical lithotriptor for common bile duct stones. Endoscopy. 14, 226-230 (1982).

Merrell SW, Schneider PD. Hemobilia-evolution of current diagnosis and treatment. West J Med. 155, 621-625 (1991).

Kim KH, Kim TN. Etiology, clinical features, and endoscopic management of hemobilia: A retrospective analysis of 37 cases. Korean J Gastroenterol. 59, 296-302 (2012).
Jones HJ, Staud R, Williams RC. Rupture of a hepatic artery aneurysm and renal infarction (two complications of fibromuscular dysplasia that mimic vasculitis). J Rheumatol. 25, 2015-2018 (1998).

Hidalgo F, Narváez JA, Reñé $M$, et al. Treatment of hemobilia with selective hepatic artery embolization. J Vasc Interv Radiol. 6, 793-798 (1995).

Abboud B, Ghossain A, Tohme C, et al. Aneurisme de l'artere hepatique traite par embolisation. J Chir. 131, 252-256 (1994).

Lal RB, Strohl JA, Piazza S, et al. Hepatic artery aneurysm. J Cardiovasc Surg. 30, 509-513 (1989). 